\title{
The double life of lentiviruses
}

The first lentiviral gene transfer vector, derived from HIV, was described in 1996 in a seminal article alluding to great promises for gene therapy (Science 272, 263; 1996). With time and design modifications, this vector also was destined to become an important laboratory tool. Ten years later, despite a backdrop of questions about the future of gene therapy, lentiviral vectors have finally reached the clinic for the first time, and developments of this laboratory tool are still going strong, as illustrated by two articles in this issue (p109, p117). The dual purpose of lentiviral vectors, as therapeutic agents on one hand and laboratory tools on the other, has allowed their sustained improvement throughout times of hardship for gene therapy-improvements that will eventually serve both applications.

Lentiviral vectors were created to harness the powerful capacity of HIV to infect cells and stably insert a stretch of genetic material into the cell genome. Their name comes from lentivirus, the subfamily of retroviruses to which HIV belongs. By the time lentiviral vectors came along in 1996, vectors derived from a mouse retrovirus of another subfamily, and referred to as retroviral vectors, had already been tested in clinical trials, generating exciting preliminary results. HIVbased vectors had more to offer, namely the capacity to infect nondividing cells. But it was clear from the onset that developing a therapeutic vector based on a human pathogen like HIV would take a long time.

Gene therapy with retroviral vectors has since gone through trials and tribulations, culminating in a brief phase of triumph in 2000, when Alain Fischer and Marina Cavazzana-Calvo in Paris successfully treated children with severe combined immunodeficiency by correcting the genetic defect in their bone marrow. But this very first gene therapy success turned sour in 2002, when two children developed leukemia as a consequence of the treatment. Since then, the treatment efficacy has been confirmed by the results of two parallel studies in London and Milan-17 of the 18 patients treated so far have reconstituted a functional immune system. But despite these successes, the tragic adverse events have dramatically reduced the pace of gene therapy research. Scientific and regulatory scrutiny have increased, companies that had invested in the promises of the field are going out of business or changing their plans as investors shy away.

If it was not for the promises of the basic research tool, researchers and funding agencies may have similarly abandoned efforts of vector design. But while the therapeutic prospects of lentiviral vectors inevitably followed the ups and downs of their retroviral cousins, their specific biological properties earned them unmatched popularity as a basic research tool.

Indeed, the ability to infect nondividing cells, as well as a certain resistance to epigenetic silencing once integrated in the host cell genome, are great assets. Lentiviral vectors have permitted the transduction of many cell types otherwise resistant to gene transfer, the generation of animal models-for example, for Parkinson and Huntington diseases by delivering mutant genes into the brain of rats-and the creation of transgenic mice by infection of fertilized eggs. In addition, HIV-based vectors, as nonpathogenic replicas of their harmful parent, have greatly facilitated the study of elements of HIV biology such as tropism, cell penetration, genome integration and transcriptional control.

So what will happen next? The future of lentiviral vectors in the laboratory is certainly wide open. The combination of efficient delivery with the emerging power of RNA interference is a huge asset for mammalian genetics. New vector designs facilitating the temporal and local control of RNA interference and gene replacement, such as the one described by Aebischer and Trono (p109), will facilitate studies in animal models. At the same time, high-throughput screens using renewable viral RNA interference libraries will be made possible by the array systems presented by Sabatini's group (p117).

As for the gene therapy aspect, the first clinical trial involving an HIV-based vector has been completed in the United States in 2005, in AIDS patients, and a follow-up trial evaluating safety and efficacy has been under way since July 2005. Meanwhile, several US and European groups are gearing up for phase I trials involving patients affected by AIDS or life-threatening genetic diseases.

The lessons from the retroviral vector trials should help choose the most suitable therapeutic applications and also design better vectors. Interestingly, the specific characteristics of lentiviral vectors may provide elements of solution, for example by permitting to minimize ex vivo manipulation of the bone marrow cells or reduce therapeutic doses. And through the troubled coming of age of a new, ambitious therapy, lentiviral vectors keep improving steadily thanks to their suitability as laboratory tools - a fruitful double life for the benefit of both vocations. 